\title{
émulations
}

\section{Laurent Di Filippo, Émilie Landais (dir.) - Penser les relations entre médias. Dispositifs transmédiatiques, convergences et constructions des publics}

\author{
Baptiste Campion
}

Émulations - Revue de sciences sociales

2018. Comptes rendus critiques. En ligne

\section{Article disponible à l'adresse suivante}

https://ojs.uclouvain.be/index.php/emulations/article/view/7473

\section{Pour citer cet article}

Baptiste Campion, «Laurent Di Filippo, Émilie Landais (dir.) - Penser les relations entre médias. Dispositifs transmédiatiques, convergences et constructions des publics », Émulations, en ligne. Mise en ligne le 18 décembre 2018.

DOI : $10.14428 /$ emulations.cr.046

Distribution électronique : Université catholique de Louvain (Belgique) : ojs.uclouvain.be

(C) Cet article est mis à disposition selon les termes de la Licence Creative Commons Attribution, Pas d'Utilisation Commerciale 4.0 International. http://creativecommons.org/licenses/by-nc/4.0/

Éditeur : Émulations - Revue de sciences sociales / Presses universitaires de Louvain https://ojs.uclouvain.be/index.php/emulations

ISSN électronique : $1784-5734$

UCL PRESSES UNIVERSITAIRES DE LOUVAIN 


\section{Laurent Di Filippo, Émilie Landais (dir.) - Penser les relations entre médias. Disposi- tifs transmédiatiques, convergences et constructions des publics}

Baptiste Campion ${ }^{1}$

Recensé : Laurent Di Filippo, Émilie Landais (dir.) (2017), Penser les relations entre médias. Dispositifs transmédiatiques, convergences et constructions des publics, Strasbourg, Néothèque, $319 \mathrm{p}$.

Dans un contexte d'évolution rapide et profonde du paysage médiatique sous l'emprise du développement des technologies numériques et, par conséquent, de transformation de la production et de la réception des médias, les notions de " convergence " médiatique, de " transmédia » et de " cross-média » apparaissent de plus en plus centrales dans l'étude des pratiques culturelles médiatisées. Ces technologies sont aujourd'hui utilisées dans bon nombre de discours à l'évidence académiques, mais aussi médiatiques, industriels ou culturels ; au risque parfois de se voir transformées en mots-valises vides de sens, que chaque acteur investirait au gré de ses projets, de la mode ou du marketing. Les recherches sur le transmédia, pour beaucoup menées dans la lignée des travaux d’Henri Jenkins (2004; 2006 ; 2009), ont pour point commun d'interroger les liens entre productions culturelles et médiatiques d'une part, et l'expérience de réception d'autre part. Mais, ce faisant, elles ont tendance à aborder en ordre dispersé des questions parfois très différentes (de conception, d'analyse du récit, de réception) sur des terrains potentiellement très variés (allant des fictions télévisuelles à la médiation muséale en passant par toute une variété de dispositifs se revendiquant, à un degré ou un autre, " ludiques ", " interactifs ", et mobilisant à ce titre divers supports).

Cet ouvrage collectif coordonné par Laurent Di Filippo et Émilie Landais constitue les actes d'un colloque intitulé « Dispositifs transmédiatiques, convergences et construction des publics » qui s'est tenu à Metz en 2012. Il a pour ambition de faire le point sur ces travaux multiples qui, parfois, s'ignorent alors qu'ils puisent aux mêmes sources théoriques. Constitué de onze contributions indépendantes, pour la plupart produites par de jeunes chercheurs (les auteurs sont en majorité doctorants ou post-doctorants), il permet de découvrir un grand nombre de recherches récentes, pour certaines encore en cours, qui convoquent chacune à leur manière les notions de convergence et de transmédia, en cohérence avec l'ambition affichée par les coordinateurs d'évaluer la portée heuristique de celles-ci dans différents champs des sciences humaines. C'est ainsi que sont abordés dans ce recueil des dispositifs aussi variés

${ }^{1}$ Institut des hautes études des communications sociales (IHECS), Bruxelles. 
que des applications éducatives, des dispositifs de médiation muséale, la mise en valeur du patrimoine, des fictions déclinées sur divers supports, des pratiques de réappropriation, des jeux sur téléphone portable ou les transformations du cinéma.

L'ouvrage s'ouvre par une longue introduction rédigée par les coordinateurs intitulée « Dispositifs transmédiatiques, convergences et publics : construire et penser les relations entre médias", qui se présente comme une discussion théorique sous la forme d'une large revue commentée de la littérature (principalement francophone), visant tout à la fois à définir les notions de transmédia et concepts liés, à en situer les différentes approches et les questions qui les caractérisent, ainsi qu'à en évaluer la portée et les limites. Ce travail se fait en trois grandes étapes. Dans un premier temps, les auteurs situent l'apparition des dispositifs transmédiatiques dans les évolutions du paysage médiatique contemporain, avec une interrogation transversale : cette convergence est-elle une conséquence des développements technologiques actuels ou constitue-t-elle une caractéristique culturelle plus large ? II s'agit ainsi de se demander dans quelle mesure les dispositifs transmédia existent et présentent des spécificités propres. Dans un deuxième temps, Laurent Di Filippo et Émilie Landais portent un regard critique sur les différentes manières par lesquelles les chercheurs envisagent la culture transmédia comme une spécificité de la culture contemporaine. Ils mettent notamment en garde contre les illusions de nouveauté et de synchronicité, arguant que les principes de convergence et d'écriture transmédiatique, et les compétences qu'ils présupposent, ont pu exister dans des temps parfois très anciens (ils citent notamment les représentations religieuses construites à la fois par assimilation et différenciation par rapport à des corpus déjà existants), même si les technologies numériques actuelles permettent de développer des processus à une échelle bien plus large. Ils en concluent que l'essentiel n'est pas d'interroger les phénomènes transmédiatiques sous l'angle de la nouveauté, mais sous celui du tournant particulier qu'ils prendraient dans notre environnement culturel numérique. Cette interrogation amène à la dernière étape de leur raisonnement. Ces approches transmédias sont actuellement très souvent cantonnées dans une analyse relativement classique des dispositifs médiatiques, sorte d'avatar transmédiatique de l'analyse littéraire, et sont très rarement enrichies par d'autres aspects (comme les processus de conception ou de réception) ou d'autres disciplines (comme l'anthropologie ou la sociologie). Les auteurs défendent la portée interdisciplinaire de cette approche, insistant notamment sur la nécessité d'un " élargissement théorique » et d'un travail de conceptualisation de termes largement employés dans des sens parfois divergents (" expérience ", " culture », etc.). Les concepts propres à une approche " transmédia » devraient être l'occasion, pour les auteurs, de remettre en question les problèmes étudiés par les sciences de l'homme et de la société pour autant qu'on dépasse la dimension parfois « prophétique » qui leur est donnée par certains auteurs phares (p. 40). On devine en creux que c'est ce qu'ils entendent montrer à travers la variété des contributions de cet ouvrage, qui se saisissent de ces concepts et cherchent à les appliquer, de manière concrète, pour comprendre les questions et enjeux qui se posent sur des terrains spécifiques et contextualisés. 
La suite de l'ouvrage est organisée en trois parties. La première, intitulée " Dispositifs transmédiatiques, convergences et publics : construire et penser les relations entre médias ", comporte des contributions s'intéressant à la manière dont des dispositifs transmédias développés dans la sphère culturelle présupposent ou impliquent une représentation et une segmentation des publics et de leurs usages. Frédéric Marty montre ainsi, dans « Les représentations du public enseignant chez les concepteurs de dispositifs numériques éducatifs. Le nouveau player du site.tv ", comment les enseignants utilisateurs d'une plateforme de vidéos éducatives sont vus comme des créateurs de contenus. Ce postulat de base amène les concepteurs de la plateforme à repenser son interface dans le but de favoriser des pratiques de réappropriation personnelle des ressources, mais de manière limitée par des facteurs externes (la nécessité de respecter les droits d'auteur liés à ces mêmes ressources). Dans son article « Interroger la notion de dispositif transmédiatique au musée d'ethnographie : le cas du Museon Arlaten ", Eva Sandri étudie la manière dont l'introduction des technologies de l'information et de la communication (TIC) dans le cadre de la rénovation d'un musée d'ethnographie amène à penser une interopérabilité et une convergence des supports de médiation muséale à destination de publics différenciés, bien plus qu'à penser ces dispositifs dans le cadre d'une expérience intégrée. Dans sa contribution portant sur " Les dispositifs de médiation éducative au Château de Versailles : Fiction, Transmédia et Multimodalité ", Julie Pasquer-Jeanne fait un constat assez similaire au départ des dispositifs de médiation éducative du Château de Versailles, mettant en avant le rôle médiateur et intégrateur des fictions utilisées, qui sont disparates et non coordonnées entre elles, mais mises en cohérence par l'Histoire et le point commun que constitue pour chacune le château. Dans " L'approche transmédiatique dans les dispositifs photo-humanitaires et l'idée de "spectateur citoyen" ", Rosana Contreras-Gama interroge la manière dont les représentations du public orientent la conception d'expositions photo-humanitaires vers des dispositifs plus ouverts favorisant l'expérience et l'engagement du spectateur, au détriment peut-être d'autres postures de visite.

La deuxième partie, intitulée " Pratiques hétérogènes parmi les publics ", interroge l'expérience proposée par des dispositifs transmédiatiques. Dans « Enjeux des dispositifs transmédiatiques comme cadres de l'expérience ", Marta Boni se base sur les cadres de l'expérience d'Erving Goffman (1974) pour envisager les dispositifs transmédias, vus comme une communication multicanale où le récepteur doit sans cesse s'interroger sur la situation médiatique qu'il est en train de vivre. Se basant sur l'analyse d'une série télévisée aux prolongements numériques, elle aboutit à une typologie des interactions des consommateurs avec l'œuvre de fiction. Dans « Prendre en compte les pratiques non contributives. Approche méthodologique de l'étude des pratiques (trans)médiatiques des lecteurs de la bédénovela Les Autres Gens ", Julien Falgas interroge ainsi les pratiques non contributives des lecteurs d'une bédénovela diffusée en ligne et ses différentes prolongations, ce qui lui permet lui permet de cartographier les pratiques des fans et des non-fans. II montre que si le dispositif est avant tout pensé pour un public contributif, une part importante des lecteurs ne se situe pas nécessairement dans cette perspective. Dans sa contribution intitulée « La transphonographie à l'essai 
du metal symphonique de Nightwish et de ses amateurs : un voyage vers la création continuée ", Cyril Brizard, enfin, s'intéresse à la pratique originale d'un groupe de metal symphonique et montre dans quelle mesure on peut la comprendre comme un ensemble de pratiques inter- et trans-phonographiques de différentes natures (fragmentation, intertextualité, citation, etc.). Ceci l'amène à considérer l'œuvre comme une série d'appropriations et restitutions de ce qui est connu et reconnu du médiateur.

La troisième partie, intitulée "Changements et résistances", comprend plusieurs contributions qui montrent que les pratiques transmédiatiques viennent avant tout ajouter une couche à des pratiques déjà existantes. Ainsi, Camille Jutant, étudiant des jeux de médiation à la culture dans son article "Pratiques transmédiatiques des publics de la culture : jeux en réalité alternée et jeux sur téléphone portable », décrit l'expérience transmédiatique comme un "surenchérissement " communicationnel : l'expérience différente naît du fait que l'utilisateur va se voir proposer plus de choses, par l'entremise de différents canaux, que ce qu'il aurait pu voir avec une communication monocanale. Dans « Envisager la cocréation dans les jeux en ligne comme média alternatif ", Patrick Prax propose ainsi un modèle d'analyse centré sur le concept de co-création qui a pour ambition de rendre compte de la manière dont les joueurs de jeux en ligne peuvent dépasser les contingences (notamment idéologiques) imposées par les éditeurs à travers la construction d'une expérience cross-médiatique composée du jeu et des productions de la communauté. Dans « Versionnement multisupport et surenchère technologique : conflits techniques et esthétiques ", Yannick Pourpour, enfin, s'intéresse à la déclinaison de productions cinématographiques dans de multiples formats, montrant comment celles-ci sont pensées comme la rencontre entre la recherche d'une " esthétique de la surenchère " (comme argument commercial destiné aux spectateurs, à qui l'on promet une expérience différente) et de choix techniques (liés aux contraintes de production ou destinés à promouvoir une technologie).

Cette richesse et cette diversité, dont témoigne ce rapide panorama des contributions, constituent le principal intérêt de l'ouvrage, mais aussi son principal défaut. L'aspect pluriel et polyphonique de l'ouvrage rend par moments une lecture transversale difficile, notamment en vue d'y saisir une cohérence globale, notamment terminologique. Ainsi, si certains contributeurs reprennent le concept de transmédia tel que défini par Jenkins, d'autres s'en distinguent explicitement, alors que d'autres encore lui préfèrent les termes de "communication multicanale » ou de "formes plurimédiatiques». Si dans le chapitre introductif les coordinateurs plaidaient pour un travail de définition commune des termes essentiels reliant les différentes approches de ce champ de recherche, force est de constater que cela n'est pas pratiqué dans la diversité des contributions, même si chaque approche questionne, à sa manière, ces termes transversaux. Sans renoncer à la diversité des approches, les contributions auraient gagné à être situées et discutées les unes par rapport aux autres dans une introduction plus spécifique, distincte de la revue plus générale de la littérature, mettant en perspective les approches des différents auteurs. 
Cette limite est particulièrement visible quand on cherche à lire l'ouvrage comme un tout plus que comme un recueil de contributions indépendantes. Cet ouvrage aurait peut-être mérité une conclusion prenant de la hauteur afin de mettre en œuvre un recul réflexif et critique sur la portée de ces concepts mobilisés sur des questions très différentes, et d'opérationnaliser l'élargissement de ces concepts au champ des sciences humaines, conformément à l'ambition affichée des coordinateurs de l'ouvrage. Le chapitre introductif, dans son vaste panorama des approches du transmédia, le fait en partie, situant les différentes contributions dans ce vaste champ de recherche, mais sans identifier ou expliciter ce qu'elles ont de spécifique au regard des enjeux heuristiques posés par les notions interrogées. Cette limite formelle n'enlève cependant rien à l'intérêt individuel de chacune des contributions. Ainsi, cet ouvrage est à recommander pour qui s'intéresse à la recherche francophone récente dans cette aire de recherche très active, à savoir les dispositifs de médiation culturelle (au sens large) et leur manière d'envisager l'expérience de réception par combinaison de différents supports médiatiques, ou par l'organisation de la cohabitation de publics aux attentes et représentations diversifiées.

\section{Bibliographie}

Goffman E. (1974), Frame Analysis: An Essay on the Organization of Experience, Boston, Northeastern University Press.

Jenkins H. (2004), "The Cultural Logic of Media Convergence ", International Journal of Cultural Studies, vol. 7, n 1, p. 33-43.

Jenkins H. (2006), Convergence Culture: Where Old and New Media Collide, New York, New York University Press.

Jenkins H. (dir.) (2009), Confronting the Challenges of Participatory Culture: Media Education for the 21st Century, Cambridge, The MIT Press. 